\title{
Public health preventive action to start from the fourth decade of life?
}

\author{
Sarah Cuschieri ${ }^{\mathrm{a}, *}$, Stephan Grech ${ }^{\mathrm{b}}$ \\ ${ }^{a}$ Department of Anatomy, Faculty of Medicine and Surgery, University of Malta, Msida, Malta \\ ${ }^{\mathrm{b}}$ Mater Dei Hospital, Msida, Malta
}

A R T I C L E I N F O

\section{Keywords:}

Type 2 diabetes mellitus

Noncommunicable diseases

Epidemiology

Age groups

Public health

\begin{abstract}
A B S T R A C T
Problem considered: Non-communicable diseases (NCDs) are a global epidemic. With the start of a new decade, new strategies and health goals are expected to be formulated. The aim was to investigate a highly prevalent NCD European state to assess the age of onset for most NCDs. Such evidence can aid in the development of new preventive strategies.

Methods: A national health examination survey was conducted to explore the adult population (18-70 years) of Malta for common NCDs. Both self-reported and examination data were used to diagnose type 2 diabetes, dyslipidaemia, hypertension, overweight and obesity. The study population was stratified by ten years agegroups and sex for both descriptive and analytic analyses. Association studies were conducted to identify links between type 2 diabetes, dyslipidaemia, hypertension, overweight and obese status and different age groups, while adjusting for confounders.

Results: The men were significantly more metabolically unhealthy that the women across all age groups. However, a higher newly diagnosed diabetes prevalence across all age groups was established for women. Dyslipidemia, diabetes and hypertension were observed to be present from the 30-39 year age group. On modelling, this age group was associated with these NCDs even after adjusting for confounders.

Conclusion: The fourth decade of life appears to be the starting point of metabolic dysregulation, contributing to long-term health and economic burdens. Furthermore, considering this is a childbearing age group, metabolic impact can be transmitted on to their offspring. Preventive action including dysglycaemia and dyslipidaemia screening should be considered from a young age.
\end{abstract}

\section{Introduction}

Non-communicable diseases (NCDs) have been on the incline for the past years. Cardiovascular disease, cancer, chronic lung disease and diabetes are the four major global NCDs. ${ }^{1-3}$ NCDs contribute to a global mortality rate that varies from around $30 \%-94 \%$, with the highest morality rates reported to affect developed countries. ${ }^{4}$ This has led to the establishment of both global and country level action plans to try to reduce the NCD epidemic by $2020 .^{5-8}$ Preventive and management plans usually target high-risk populations and adults within their fifth decade of life. , $9^{-1}$

Malta, an island state in the middle of the Mediterranean Sea, has been reported to be a non-communicable diseases hub, where NCDs result in $90 \%$ of the mortality rate of the country. ${ }^{4}$ It has been reported that this country has the highest rates of type 2 diabetes and obesity when compared to neighbouring European countries. ${ }^{10-12}$ The new decade, twenty-twenties, brings with it the onset of new strategies and goals targeting numerous NCDs. Considering the high prevalence of
NCDs, it was considered as appropriate to investigate this population. The aim was to explore for potential trends and associations with NCDs across different age groups and hence provide evidence-based data that can be used for new preventive strategies and goals.

\section{Method}

\subsection{Data sampling and collection}

A national representative sample population was obtained from a national register by undergoing a random single stage stratification by age (18-70 years), sex and locality. Since the geographical area of Malta is small, a single stage stratification is appropriate. A sample population of 4000 adults representing approximately $1 \%$ of the country's total adult population by age and sex was obtained. All individuals living in Malta for at least 6 months were eligible, irrelevant of their country of origin. Pregnant women, those too ill to attend the examination hub and those living abroad but still registered in the local

\footnotetext{
* Corresponding author.

E-mail address: sarah.cuschieri@um.edu.mt (S. Cuschieri).
} 


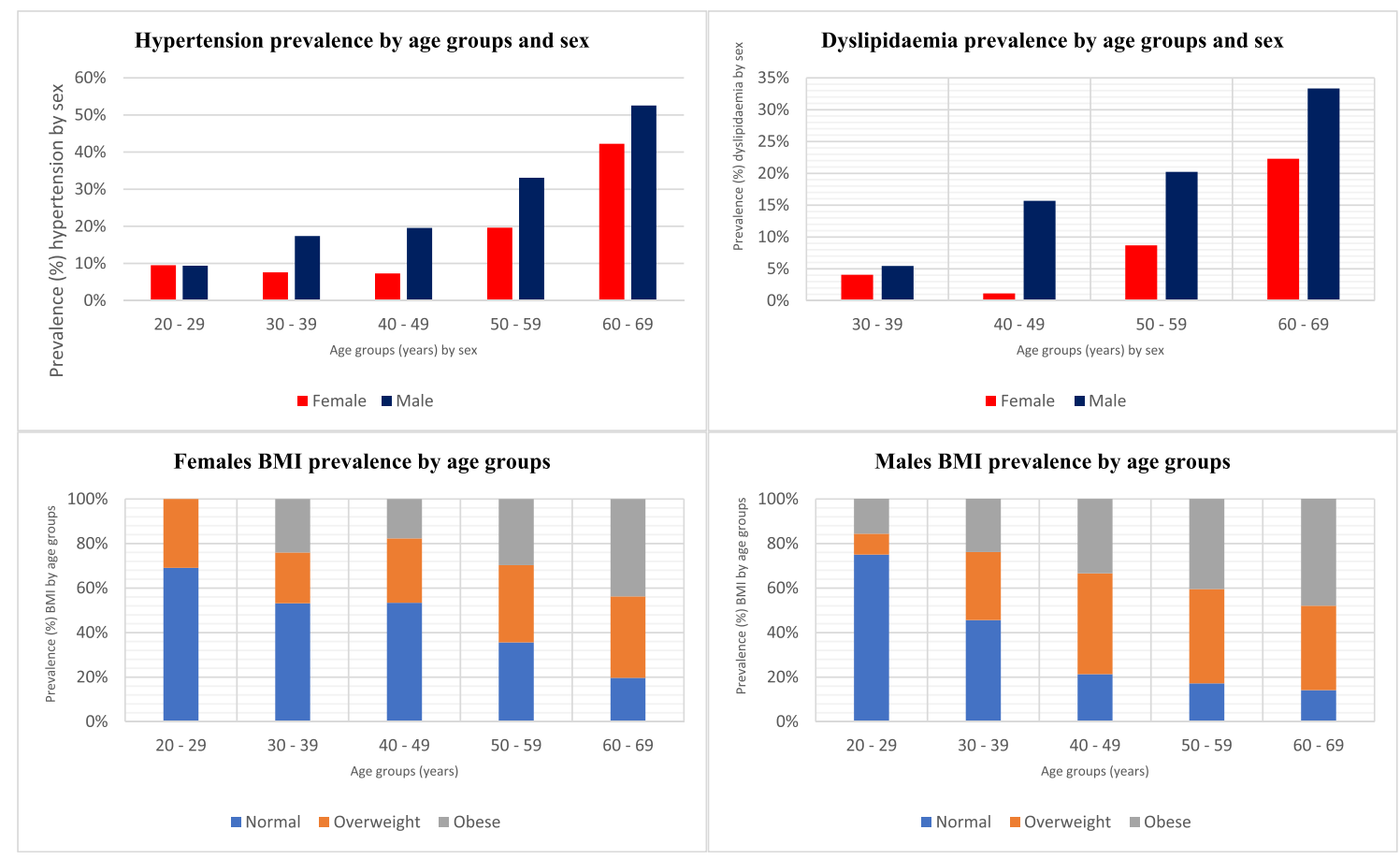

Fig. 1. Illustrates the metabolic profile affecting the different age groups by sex.

registry were excluded.

A health examination survey was conducted by setting up an examination hub in each town. The detailed protocol has been described elsewhere. ${ }^{13}$ In brief, a validated questionnaire was utilized to collect data on sociodemographic status, highest education level and medical related data. The examination consisted of blood pressure, weight, height, waist and hip circumference measurements. Blood samples for fasting blood glucose (FBG) and lipid profile were also collected. The blood testing was performed in an accredited lab. Ethical and data protection approvals were granted from the University of Malta Research Ethical Committee (UREC) and the Information and Data protection national commissioner respectively. Participants gave their written informed consent prior to engaging in the health examination survey.

\subsection{Definitions}

Naïve dyslipidaemia was defined as those participants who had a confirmed measurement of a high LDL-C, high triglycerides and low HDL-C and not on any medication. Hypertension was labelled in those participants who on examination had a high systolic $(\geq 140 \mathrm{mmHg}$ ) or diastolic blood pressure $(>-90 \mathrm{mmHg}$ ) or gave a history of hypertension or were on anti-hypertensive medication. The definition for body mass index (BMI) followed the WHO criteria, where BMI of $<24.99$ $\mathrm{kg} / \mathrm{m}^{2}$ was labelled as normal; $25-29.99 \mathrm{~kg} / \mathrm{m}^{2}$ as overweight and $\geq 30 \mathrm{~kg} / \mathrm{m}^{2}$ as obese. ${ }^{14}$ Impaired fasting glucose (IFG) was defined as the presence of FBG levels between $5.60 \mathrm{mmol} / 1$ and $6.99 \mathrm{mmol} / 1$ without the presence of impaired glucose tolerance. ${ }^{15}$ Participants reporting a previous diagnosis of diabetes mellitus or else on diabetic treatment were classified as previously known diabetic. Subjects with an FBG measurement $\geq 7 \mathrm{mmol} / \mathrm{L}$ and not previously known diabetic were labelled as as newly diagnosed diabetic. ${ }^{5}$ Global diabetes was the combination of both diabetes sub-categories.

\subsection{Data analysis}

The responders were statistically weighted using IBM SPSS software in order maintain representation of the population. The adjusted population was then analysed by stratifying the population by sex (men and women) and ten-year age groups. Both descriptive and analytic analyses were performed for anthropometric, biochemical and socioeconomic variables. Comparative categorical analyses between the different socio-economic factors (highest education level and locality), lifestyle habits (smoking and alcohol), presence of hypertension, diabetes and obesity utilising the chi square test for each sex category and age group was done. Continuous analysis was performed through an independent student t-test. A $p$-value of $<0.05$ was considered as statistically significant. Modelling was performed through binary multiple logistic regression analyses to establish any potential associations between different conditions (diabetes, hypertension, overweight status, obesity status and dyslipidaemia) and the different ten-year age groups while adjusting for potential confounding factors (sex, locality, education level, occupation, anti-hypertensive medication, dyslipidaemic medication, family history of cardiovascular disease, family history of type 2 diabetes, smoking and alcohol habits).

\section{Results}

\subsection{Sociodemographic data}

This national representative study achieved a response rate of $47.15 \%$ with a total adjusted population of 3947 adults (male $n=1988$ ). The response rate did not differ across the ten years age groups and sex $(p=0.12)$. Across the different age groups, the male population showed no significant difference between their residing district ( $p=0.34$ ) and age groups. Conversely, the female population exhibited a significant difference $(p=0.04)$, with the 30 to 39 -year age group mostly residing in the Southern Harbour while the rest of the age groups mostly residing in the Northern Harbour. Of note, out of all the different age groups and sex categories, the 30 to 39 -year age group reported achieving the highest university level education (Female 39.49\%; Male 43.78\%).

\subsection{Metabolic profile}

The median biochemical profile (FBG and lipid profile) fell within 


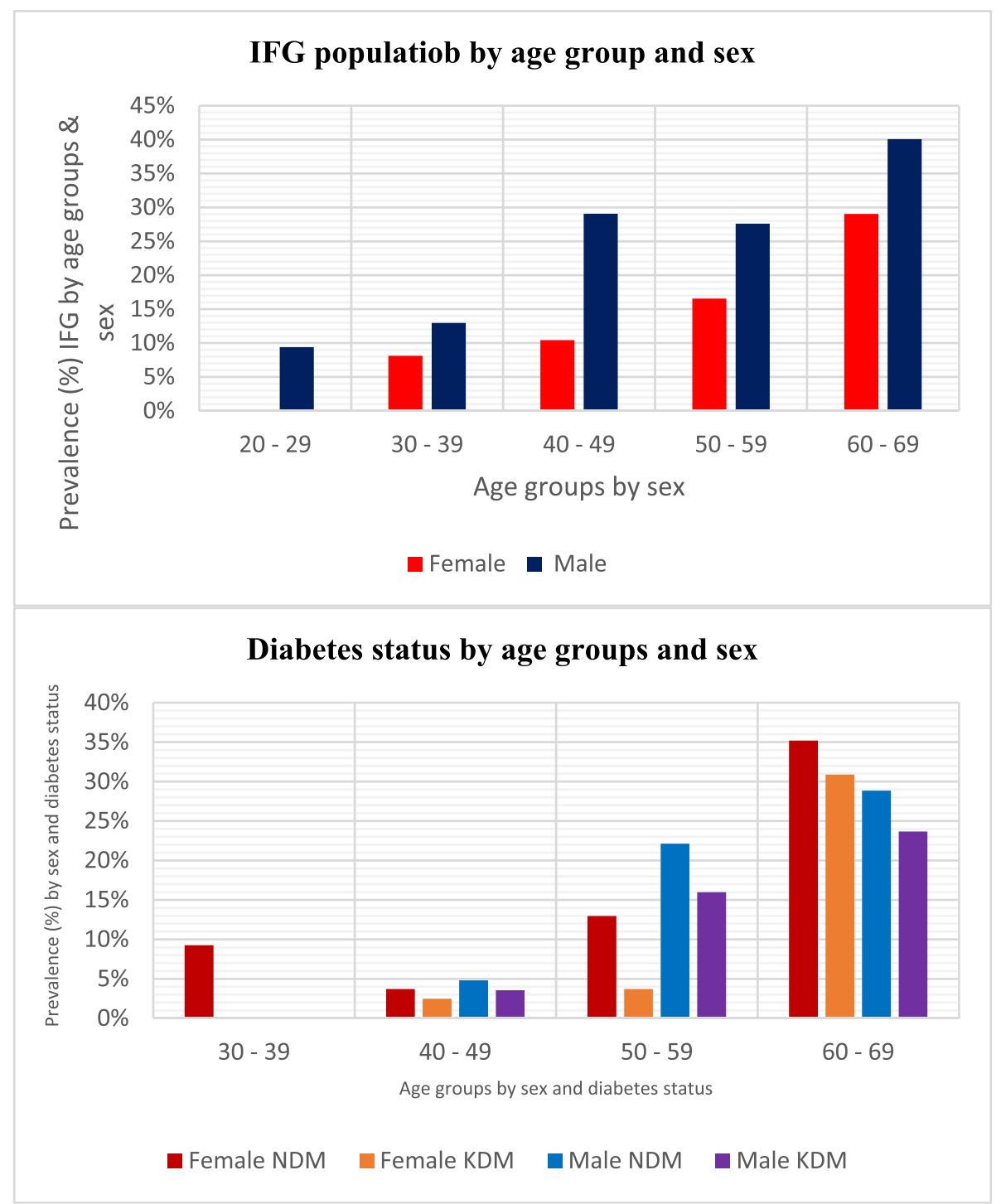

Fig. 2. Illustrates the dysglycaemic profile affecting the different age groups by sex.

the normal laboratory specific ranges across all the different age groups. However, overall the male population had significantly higher median values than their female counterparts ( $p=<0.01$ respectively).

Various metabolic conditions were present from the 30 to 39 -year age group (Fig. 1). Naïve dyslipidaemia (high LDL-C, high triglycerides and low HDL-C) predominated this age group. None of these participants reported to be on any dyslipidaemia medication but were found to have high dyslipidaemia levels on examination.

Hypertension was recorded across all the age groups. However, a drastic rise in hypertension prevalence was observed in the male population between the 20 to 29-year and the 30 to 39-year age groups ( $p=<0.01$ ). $25.37 \%$ of the $30-39$ year group (CI 95\%: $16.41-37.01$ ) reported to be on anti-hypertensive medication. The male hypertension predominance continued as the age progressed, as seen in Fig. 1.

The male population appeared to have a rapid decline in normal BMI status as the age groups progressed. While an overweight and obese status showed progressive prevalence increase from the 30 to 39 year age group (Fig. 1). Although the female population followed a similar pattern, the proportion of obese status affecting the 30 to 39 year age group was higher than the 40 to 49-year age group. Conversely, the proportion of females within the overweight status was higher within the 20 to 29 -year age group rather than both the 30 to 39 year and 40 to 49-year age groups (Fig. 1).

\subsection{Dysglycaemia profile}

The men exhibited an impaired glucose regulation (IFG) status as early as the third decade while a proportion of the female population were diagnosed with new onset of diabetes from the fourth decade, as seen in Fig. 2. IFG appeared to be male predominant across all age groups (Fig. 2). The women exhibited a higher proportion of newly diagnosed diabetes across all age groups, as seen in Fig. 2. Of note, a family history of type 2 diabetes did not predominate within both sexes (Female 8.47\% CI 95\%: 7.31-9.79; Male 8.31\% CI 95\%: 7.17-9.60).

\subsection{Lifestyle habits}

Men reported higher smoking and alcohol consumption habits than the women. The men within the 30 to 39 -year age group reported the highest smoking and alcohol consumption habit prevalence when compared to the other age groups, as seen in Fig. 3. Similarly, among the women, the 30 to 39-year age group reported the highest alcohol habit, however this was not the case for smoking, as seen in Fig. 3.

\subsection{Modelling}

On performing multiple binary logistic regression modelling, the 30 


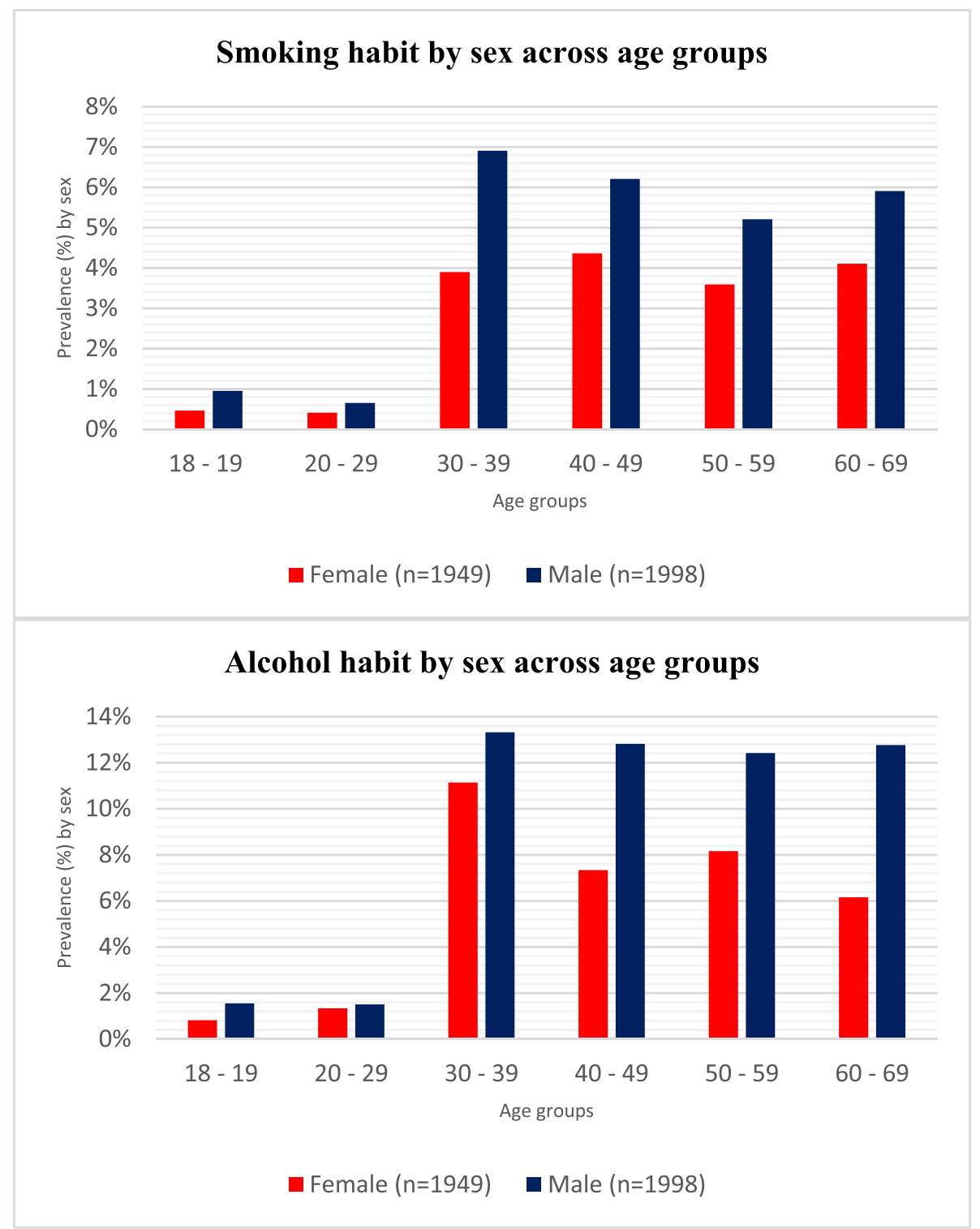

Fig. 3. Illustrates the distribution of smoking and alcohol habits by sex and age groups.

to 39-year age group (when compared to the 70 year age group), was found to associated with type 2 diabetes (OR: 1.06 CI 95\%: 1.02-1.21 $p=<0.01$ ), with hypertension (OR: 1.16 CI 95\%: $1.05-1.55$ $p=<0.01$ ) and with overweight status (OR: 1.21 CI 95\%: 1.11-1.40 $p=<0.01$ ) on adjusting for sex, locality, education level, occupation, anti-hypertensive medication, dyslipidaemic medication, family history of cardiovascular disease, family history of type 2 diabetes, smoking and alcohol habits. Furthermore, the 30 to 39-year age group (when compared to the 70-year age group) was initially found to be associated with dyslipidaemia (OR: 67.59 CI95\%: 16.40-78.64 $p=<0.01$ ). However, this association was lost on adjusting for confounders $(p=0.41)$.

\section{Discussion}

The onset of metabolic abnormalities including dysregulation of plasma glucose and dyslipidaemia were observed from the fourth decade of life. In fact, alarmingly almost one-tenth of the female population within this age group were diagnosed with new onset of diabetes. These glycaemic changes have a major health impact later on in life especially in the development of cardiovascular diseases. ${ }^{16}$
Furthermore, should these women become pregnant, their offspring is more likely to be large for gestational age with a high adiposity risk across the life course. ${ }^{17}$ The men appear to have a higher predisposition for atherosclerosis, with an abnormal metabolic profile and an unhealthy lifestyle. This provides a higher cardiovascular risk, greater loss of life years and mortality risk. ${ }^{16}$

A high body mass appears to present among the majority of the 30 to 39-year age group in both sexes. This might be the underlying cause for the development of early onset diabetes, dyslipidaemia and hypertension. In fact, the metabolic syndrome is defined as the presence of obesity along with other metabolic factors including the aforementioned. ${ }^{19-22}$

The 30-39 year age group is a precarious one, since it is the new generation typical child-bearing period. Parental metabolic changes can be passed on to the offspring. It has been reported that the epigenetic effect of paternal obesity is transferred to their offspring while maternal dysglycaemia and obesity is also transmitted to the offspring. ${ }^{23}$ Therefore, the offspring is at higher metabolic risk and susceptibility to develop non-communicable diseases, leading to an on growing population and economic burden.

Interestingly, the fourth decade subgroup reported the highest 
education level, up till university level, when compared to the rest of the age groups. Hence, this age group is considered to be well equipped with knowledge and understanding of health risk factors. However, these were generally observed to lead an unhealthy lifestyle with presence of metabolic abnormalities, which goes against the literature. ${ }^{18}$

Environmental factors might be leading to the present relationships and observations between the 30-39 year age group and different metabolic abnormalities It has already been noted that a change in dietary behaviour has occurred in the Maltese Islands, with a change from a Mediterranean diet to a more Westernized diet. ${ }^{24}$ It was noted that the food environment, like in other countries, including within high-educational institutions, is bombarded with high sugar, fat and salt food. ${ }^{25,26}$ Furthermore, it has been reported that low vegetable and fruit intake along with the high intake of sugar and salt resulted in one in five deaths in 2017 among the Maltese population. ${ }^{27}$ It is also a cultural norm in Malta to indulge in a large meal portion size as well as the tendency to engage in feasts and celebrations with abundance of food and drink typically high in saturated fats and sugars. ${ }^{28,29}$

\subsection{Study limitations}

The strengths of this study were that it was a national representative study performed in a highly prevalent NCD country. Fieldworkers underwent revalidation and training sessions every couple of months to ensure validity and standardization throughout the data collection. None of the examined measurements were rounded up but the actual reading was recorded and hence this eliminated digital preference. However, this was a cross-sectional study and hence unable to assess for temporal relationships. Potential non-response bias could have been present that effected the results. Even though this was a national representative study, a small sample size was present and subject to type 2 errors with a potential effect on the study power. This study covers only the adult population between the ages of 18-70 years. Validated tools of measurement were used however, the demographic data, lifestyle habits, medical histories and family histories were all inevitably compiled via self-reporting by participants. Such data carried the risk of human bias or recall bias. Further research, ideally through a cohort study should be performed to validate the relationships between the age group in question to the various NCDs.

\section{Conclusion}

The fourth decade of life appears to be the starting point of metabolic dysregulation within the Maltese population. This can lead to long-term health and economic burden with potential metabolic impact on their offspring. Preventive action including dysglycaemia and dyslipidaemia sceening may be considered from a young age. Targeting this age group would have a preventive impact on the life course of the adult as well as on the new offspring generation.

\section{Funding}

Funding was obtained from the University of Malta, Alfred Mizzi Foundation and Atlas Insurance (Malta). Funding had no role in the design of the study, analysis, and interpretation of data and in writing the manuscript.

\section{Declaration of competing interest}

The authors declare no conflict of interest.

\section{Acknowledgements}

The authors are extremely grateful for the strong support forthcoming from the University of Malta (through the Medical School and
Research Innovative Development Trust department) and from the Alfred Mizzi Foundation as major sponsors, as well as that of a host of others, including Atlas Health Insurance (Malta). The in-kind support and encouragement of the Parliamentary Secretariat for Health of the Government of Malta is also gratefully acknowledged. Furthermore, a note of appreciation and acknowledgement is forwarded to Professor Julian Mamo, Professor Josanne Vassallo and Professor Neville Calleja for their continuous support and advise during the academic progression.

\section{References}

1. World Health Organization. Assessing National Capacity for the Prevention and Control of Noncommunicable Diseases Global Survey. Geneva. 2016; 2016http://apps.who.int/ iris/bitstream/10665/246223/1/9789241565363-eng.pdf, Accessed date: 3 February 2018.

2. Hunter DJ, Reddy KS. Noncommunicable diseases. $N$ Engl J Med. 2013;369:1336-1343.

3. Kim HC, Oh SM. Noncommunicable diseases: current status of major modifiable risk factors in Korea. J Prev Med Public Health. 2013;46:165-172.

4. World Health Organization (WHO). Noncommunicable Diseases Progress Monitor 2017 2017; 2017https://apps.who.int/iris/bitstream/handle/10665/258940/ 9789241513029-eng.pdf?sequence=1, Accessed date: 31 December 2019

5. World Health Organization. Global Action Plan for the Prevention and Control of Noncommunicable Diseases 2013-2020. World Heal Organ; 2013:102.

6. Superintendence of Public Health. A Healthy Weight for Life: A National Strategy for Malta. 2012; 2012

7. World Health Organization. Global Strategy for the Prevention and Control of Noncommunicable Diseases. 2000; 2000http://apps.who.int/gb/archive/pdf_files/ WHA53/ea14.pdf?ua =1, Accessed date: 10 February 2018.

8. Parliamentary Working Group on Diabetes. Diabetes: A National Public Health Priority. Proposal for a National Strategy for Diabetes 2015 - 2020. Malta. 2015; 2015.

9. American Diabetes Association. Classification and diagnosis of diabetes: standards of medical care in diabetesd 2020. Diabetes Care. 2020;43:S14-S31.

10. Cuschieri S, Vassallo J, Calleja N, et al. The diabesity health economic crisis-the size of the crisis in a European island state following a cross-sectional study. Arch Publ Health. 2016;74:52.

11. Cuschieri S, Vassallo J, Calleja N, et al. Prevalence of obesity in Malta. Obes Sci Pract 2016;2:466-470

12. Cuschieri S, Mamo J. Malta: Mediterranean diabetes hub - a journey through the years. Malta Med J; 26

13. Cuschieri S, Vassallo J, Calleja N, et al. Diabetes, pre-diabetes and their risk factors in Malta: a study profile of national cross-sectional prevalence study. Glob Heal Epidemiol Genom. 2016;1. https://doi.org/10.1017/gheg.2016.18 Epub ahead of print.

14. World Health Organization. Obesity : Preventing and Managing the Global Epidemic : Report of a WHO Consultation. World Health Organization; 2000.

15. American Diabetes Association. Classification and diagnosis of diabetes: standards of medical care in diabetes 2018. Diabetes Care. 2018:41:13-27.

16. Sattar N, Rawshani A, Franzén S, et al. Age at diagnosis of type 2 diabetes mellitus and associations with cardiovascular and mortality risks. Circulation. 2019:139:2228-2237.

17. Perng Wei, Oken Emily, Dabelea D. Developmental overnutrition and obesity and type 2 diabetes in offspring. Diabetologia. 2019;62:1779-1788.

18. Zajacova A, Lawrence EM. The relationship between education and health: reducing disparities through a contextual approach. Annu Rev Publ Health. 2018;39:273-289.

19. Alberti KGMM, Zimmet P, Shaw J. Metabolic syndrome-a new world-wide definition. A consensus statement from the International Diabetes Federation. Diabet Med. 2006;23:469-480.

20. Singh O, Gupta M, Khajuria V. Lipid profile and its relationship with blood glucose levels in Metabolic Syndrome. Natl J Physiol Pharm Pharmacol. 2015;5:134.

21. Després J-P, Lemieux I. Abdominal obesity and metabolic syndrome. Nature. 2006;444:881-887

22. Eckel RH, Grundy SM, Zimmet PZ. The metabolic syndrome. Lancet. $2005 ; 365: 1415-1428$.

23. Huebschmann AG, Huxley RR, Kohrt WM, et al. Sex differences in the burden of type 2 diabetes and cardiovascular risk across the life course. Diabetologia. 2019;62:1761-1772.

24. Tessier S, Gerber M. Factors determining the nutrition transition in two Mediterranean islands: Sardinia and Malta. Publ Health Nutr. 2005;8:1286-1292.

25. Cauchi D, Rutter H, Knai C. An obesogenic island in the Mediterranean: mapping potential drivers of obesity in Malta. Publ Health Nutr. 2015;18:3211-3223.

26. Cuschieri Sarah, Camilleri Ryan, Grech S. University food environment, an example of health inequality? Malta Med Sch Gaz. 2019;3:24-28.

27. Institute for Health Metrics and Evaluation (IHME). Global Health Data Exchange. Seattle. 2018; 2018.

28. Malta Standards Authority. National Food Consumption Survey. Valletta. 2010; 2010

29. Formosa C, Savona-Ventura C, Mandy A. Cultural contributors to the development of diabetes mellitus in Malta. Int J Diabetes Metabol. 2012;20:25-29. 